\title{
Evaluation of an Orally Administered Multistrain Probiotic Supplement in Reducing Recurrences Rate of Bacterial Vaginosis: A Clinical and Microbiological Study
}

\author{
Filippo Murina*, Franco Vicariotto \\ Lower Genital Tract Disease Unit, V. Buzzi Hospital, University of Milan, Milan, Italy \\ Email: *filippomurina@tin.it
}

How to cite this paper: Murina, F. and Vicariotto, F. (2019) Evaluation of an Orally Administered Multistrain Probiotic Supplement in Reducing Recurrences Rate of Bacterial Vaginosis: A Clinical and Microbiological Study. Advances in Infectious Diseases, 9, 151-161.

https://doi.org/10.4236/aid.2019.93011

Received: April 15, 2019

Accepted: July 6, 2019

Published: July 9, 2019

Copyright $\odot 2019$ by author(s) and Scientific Research Publishing Inc. This work is licensed under the Creative Commons Attribution International License (CC BY 4.0).

http://creativecommons.org/licenses/by/4.0/

\begin{abstract}
Background: Bacterial vaginosis (BV) is the most common urogenital disease in women, affecting about $19 \%-24 \%$ of them in reproductive ages annually and after treatment, a single recurrence or more may occur in up to $58 \%$ of women within 12 months. Objective: The aim was to evaluate the effectiveness of a new orally administered food supplement, containing different probiotic strains, on women of childbearing age after the antibiotic treatment when compared with no probiotic intake. Methods: A prospective study was undertaken on 62 patients with BV. All patients were cured with metronidazole vaginal formulations ( $5 \mathrm{~g}$ of $0.75 \%$ gel once daily for 5 days or $500 \mathrm{mg}$ ovules once daily for 7 days), then after was offered the option of using a new an orally administered food supplement containing: Lactobacillus plantarum PBS067, Lactobacillus rhamnosus LRH020 and Bifidobacterium animalis lactis BL050, with a total viability of $3 \times 10^{9} \mathrm{CFU} /$ capsule (Intimique ${ }^{\circledR}$ Femme). Among these women, 50 accepted to use the new orally food supplement, while 25 patients decided to use only metronidazole (control group). Results: The recurrence rate of BV after treatment with Intimique ${ }^{\circledR}$ Femme was about $16 \%$, compared to $40 \%$ in the control group. The incidence of abnormal vaginal microbiota decreased in both groups, but it was significantly higher in the Intimique ${ }^{\circledR}$ Femme group at the end of treatment. Conclusion: This study showed that, in case of BV diagnosis, the complementary treatment of a strain-specific probiotic complex after antibiotics prophylaxis, is mandatory to reduce potential recurrences and cyclic use of further antibiotics.
\end{abstract}

\section{Keywords}

Bacterial Vaginosis, Recurrent Vaginitis, Biofilm, Lactobacillus 


\section{Introduction}

Bacterial vaginosis (BV) is the most common urogenital disease in women, affecting about $19 \%-24 \%$ of them in reproductive ages annually [1]. BV is defined as a condition characterized by the replacement of the normal vaginal flora with a high numbers of anaerobic, aerobic, and microaerophilic microorganisms. BV can best be described as an "ecological disaster" of the vaginal microflora. The normal flora, which is predominantly composed of lactobacillary morphotypes, is replaced by very high number of mainly aerobic bacteria [2]. Although at least $50 \%$ of infected women have no symptoms, the most common complaints are an abnormal vaginal discharge and a fishy odor, and there is an association with endometritis, increased risk of acquiring STI and preterm birth [3]. The etiology of bacterial vaginosis has not been fully established. The disease mechanism relates to the formation of bacterial biofilms, which are colonies of microorganisms that attach the vaginal epithelium and cover it completely or partially. Such biofilms are often resistant to antibiotic therapy, they raise the $\mathrm{pH}$, and displace endogenous flora [4]. Metronidazole (oral or topical), tinidazole (oral), and clindamycin (oral or topical) are all recommended as initial treatments offering equivalent efficacy. Nevertheless, after treatment, a single recurrence or more may occur in up to $58 \%$ of women within 12 months [5].

However, it is well known that the pathogenesis of the disease is associated with the presence of several microorganisms, such as high number of anaerobic Gram-negative, aerobic and microaerophilic microorganisms, together with a dramatically depletion in Lactobacillus count [6].

Lactobacilli may form beneficial biofilms where they produce surfactant and synthesize hydroxyl radicals and peptides that have antibacterial properties. In addition, lactobacilli can adhere to biofilms synthesized by pathogens, and some species of Lactobacillus can help destroy pathogenic biofilms. Clinical observations in recent years have prompted a turn to probiotics containing lactobacilli as a second-line treatment, as part of a multi-drug treatment. In this sense, they can represent a method of treating patients due to their ability to destroy biofilms produced by pathogens, keep vaginal $\mathrm{pH}$ in the normal range (no more than 4.5), and enable colonization resistance. Treatment does not just eradicate pathogens, but provides long-term support for a normal vaginal microbiota [7]. The aim of this work was to evaluate the effectiveness of a new orally administered food supplement, containing different probiotic strains, on women of childbearing age after the antibiotic treatment when compared with no probiotic intake. Particular attention has been paid to the long-term results of treatment and recurrences prevention.

\section{Methods}

A prospective study was undertaken on a group of 75 patients presenting, during a 12-month period of observation, recurrent BV. The trial received ethical approval from the local ethics committee and all participants provided written informed consent. 
The study included 62 patients with BV. Eligible participants were 18 50 -year-old European women who menstruated regularly and had histories of recurrent BV. They were diagnosed based on the Amsel criteria [8] in the 6 - 9 days before baseline and all cured with metronidazole vaginal formulations ( $5 \mathrm{~g}$ of $0.75 \%$ gel once daily for 5 days or $500 \mathrm{mg}$ ovules once daily for 7 days).

The exclusion criteria were: pregnancy, menopause, clinical history of hypersensitivity known to one of the components and use of anti-microbial or probiotic therapies during the previous month. Prior to any study procedure, each patient was informed about the nature and purpose of the trial, the benefits and risks. All were offered the option of using a new an orally administered food supplement containing: Lactobacillus plantarum PBS067, Lactobacillus rhamnosus LRH020 and Bifidobacterium animalis lactis BL050, with a total viability of $3 \times 10^{9} \mathrm{CFU} /$ capsule (Intimique ${ }^{\circledR}$ Femme). Among these women, 50 accepted to use the new orally food supplement after the antibiotic administration, while 25 patients decided to use only metronidazole and they were considered as a control group. The study was conducted as follows:

- Visit I: enrollment of BV diagnosed women $(n=75)$ and metronidazole treatment as above descripted;

- Visit II: verification of antibiotic therapy success and allocation to active group (Intimique ${ }^{\circledR}$ Femme) of 50 women and control group of 25 women without any further therapy;

- Reintegration period: supplementation with Intimique ${ }^{\circledR}$ Femme 1 dose/day for 14 consecutive days in the active group;

- Observation and Maintaining period: supplementation of Intimique ${ }^{\circledR}$ Femme 1 dose/day for 7 days per month from the subsequent month, after menstrual period, lasting 4 months;

- Visit III: scheduled at the end of the study or occurring during intervention period if the subject show BV symptoms;

- For safety reasons, the Investigator had the opportunity to decide of stopping the administration of the tested food supplement or to administer different therapies if necessary for the health of patients.

The primary outcome was the recurrence of BV identified based on the Amsel criteria [9], measured at the baseline and at the final visit; a positive diagnosis of BV required that three of the following four criteria were met:

- A vaginal $\mathrm{pH}$ greater than $\mathrm{pH} 4.5$;

- Proportion of clue cells $\geq 20 \%$ of total epithelial cells in the vaginal fluid;

- Presence of white and thin vaginal discharge;

- Fishy smell at whiff test.

The primary outcome was measured at the baseline and at the final visit for all patients.

Regarding the recurrence rate, the patients were subcategorised as follows:

- Responder $=$ no episode of BV relapses during the 5 consecutive months to antibiotic therapy;

- Partial responder $=$ no more than 1 episode during the 4 months observation 
period;

- Non responder $=$ more than 1 recurrence during the observation period;

- Drop-Out: relapse event during the reintegration period (initial 14 days of probiotics administration) or/and abandon of the trial by the subjects for personal or compliance reasons.

The secondary outcome was the Lactobacillus-dominated vaginal microbiota assessed by an optical microscopy at the baseline and at the final visit to evaluate the rate of its recovery [10] after the Intimique ${ }^{\circledR}$ Femme administration.

The safety was evaluated by collecting and analyzing the adverse events during the study period and by a global assessment of safety performed by Investigators.

The sample size was calculated with the assumption that previous studies have documented: recurrence rates of about $40 \%$ - 50\% within 3 months after the end of treatment [9] [11]; thus, this recurrence rate was expected in subjects not using the probiotic, while the active group was expected to experiment a reduction of recurrence rate to $\leq 25 \%$. Under these assumptions, considering a $95 \%$ confidence level and $80 \%$ power, the minimum sample size was determined to be 39 patients who successfully completed the clinical study. In recognition of several factors that may reduce the sample size, the screening group was increased to about 50 participants.

\section{Results}

The safety analysis included 75 participants in the intent-to-treat population (50 Intimique ${ }^{\circledR}$ Femme group and 25 control group with only metronidazole), that complied with the recommended regimen and completed treatment. No adverse events related to the use of the probiotic product occurred. Relapse rates based on clinical and microbiological evaluation showed a significant difference between Intimique ${ }^{\circledR}$ Femme group and control group. In the analysis of the primary objective of the study, at the final visit (Visit III) the recurrence rate of BV after treatment with Intimique ${ }^{\circledR}$ Femme was about $16 \%$, compared to $40 \%$ in the control group (Figure 1).

A statistically significant difference $(\mathrm{p}<0.001)$ was evidenced by comparing these values with those reported in the medical literature about recurrences rate data $(40 \%$ - 60\%) [12].

The comparative analysis of Amsel criteria confirmed a uniform improvement of all the examined parameters (Figure 2).

In both study groups, the incidence of abnormal vaginal microbiota decreased at subsequent visits, but the incidence of microbiologically confirmed was significantly higher in the Intimique ${ }^{\circledR}$ Femme group (Figure 3 and Figure 4 ) at the end of treatment.

Drop-out cases were recorded in both groups but in much higher amount for women included in the negative control arm.

\section{Discussion}

Depletion of commensal Lactobacillus spp., increase in bacterial diversity and 


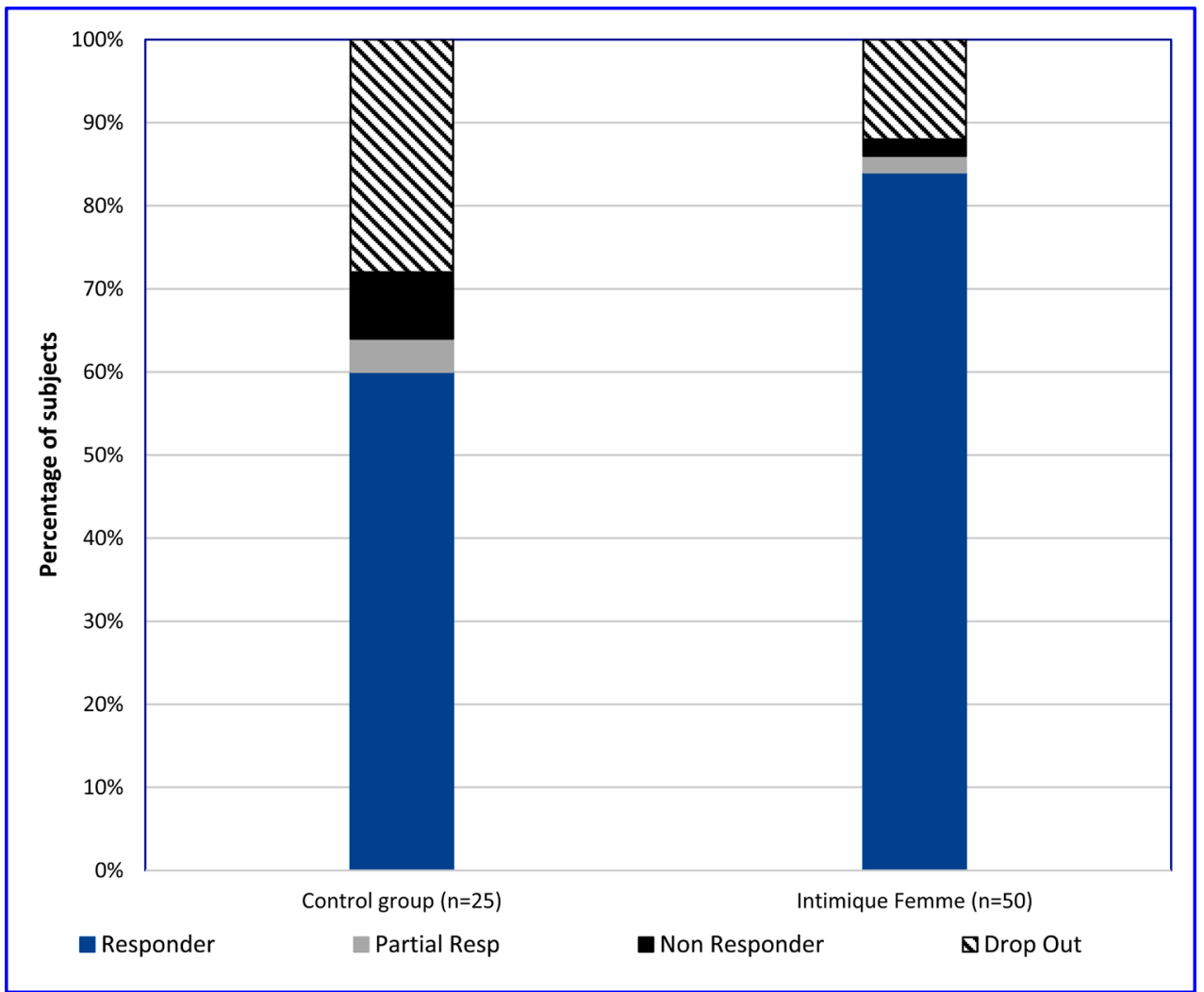

Figure 1. Final study outcome.

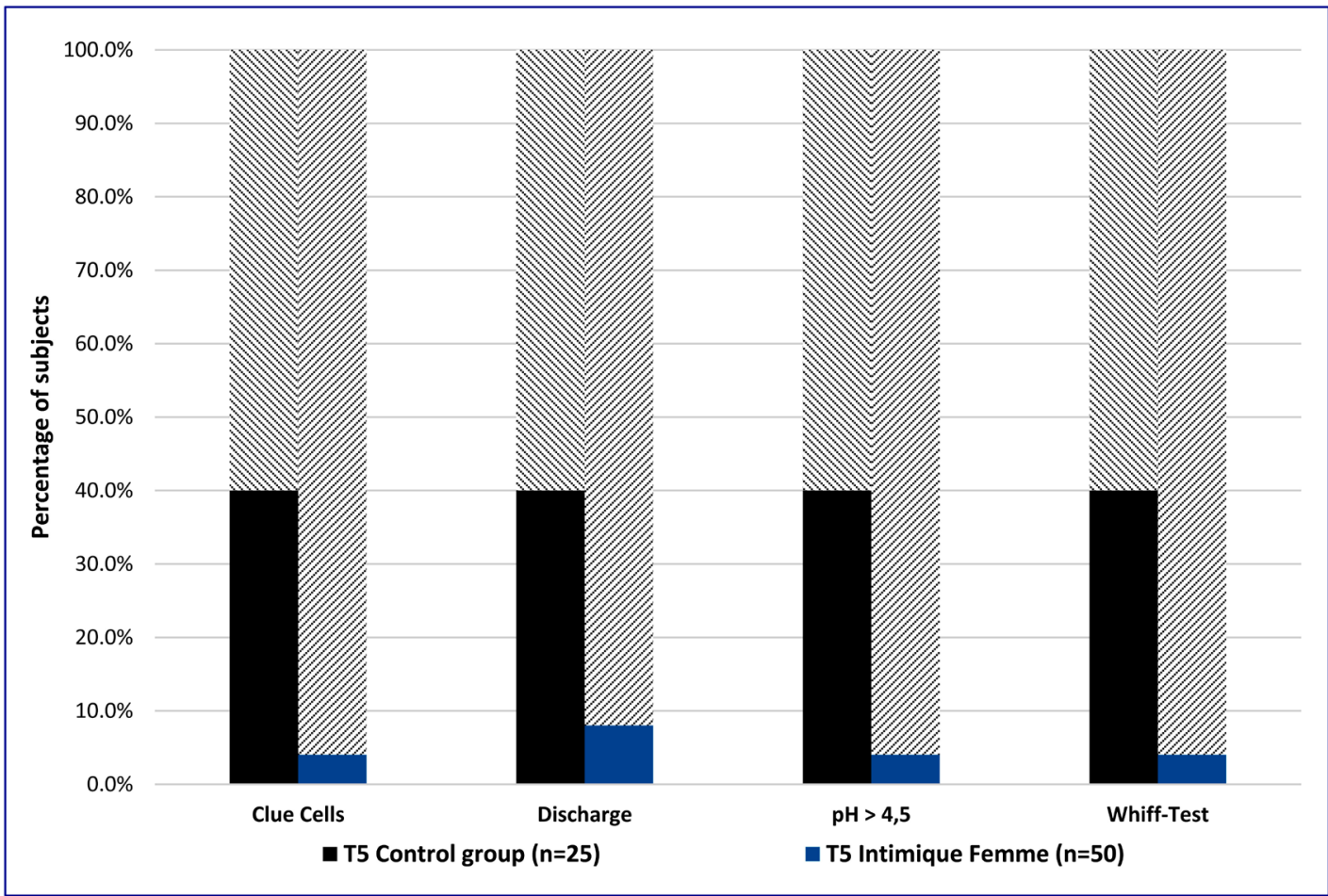

Figure 2. Percentages of subjects presenting the four Amsel's criteria for BV diagnosis after five months from antibiotic treatment. Gray bars refer to control group, treated only with antibiotic. Blue bars refer to the active group which complemented the antibiotic therapy with the probiotic supplementation. 


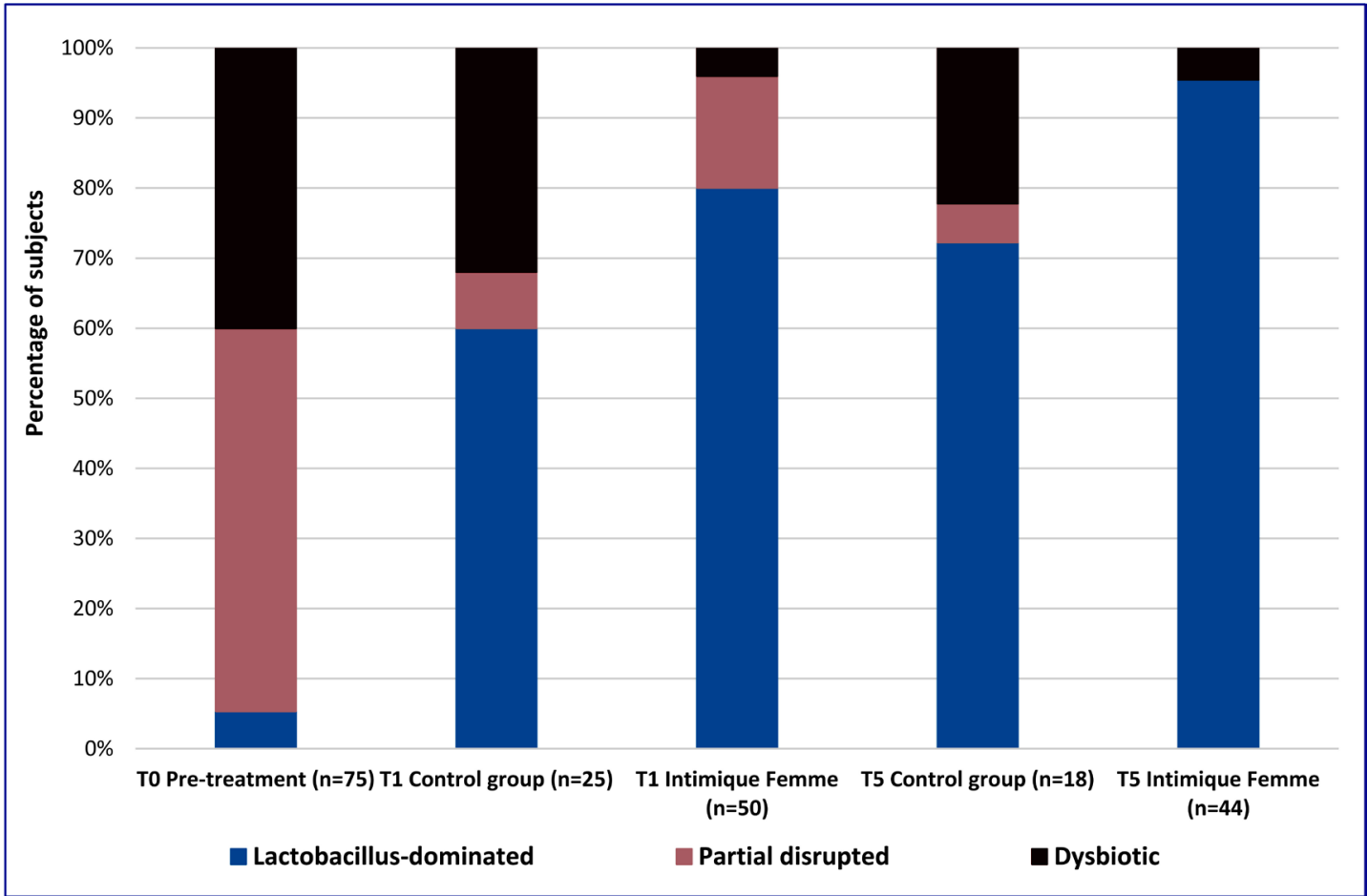

Figure 3. Vaginal wet mount microscopy examination. Evolution of the microbiota composition from pre-treatment to one month after antibiotic therapy and to five months after antibiotic in both control and active groups. Blue bars show the in-group relative percentage of subjects presenting a Lactobacillus dominated vaginal microbiota. Pink bars refer to a partial disrupted microbiota (presence of both lactobacilli and Gram-negative bacteria). Red bars refer to dysbiosis condition (depletion of vaginal lactobacilli in favor of BV-associated bacteria).

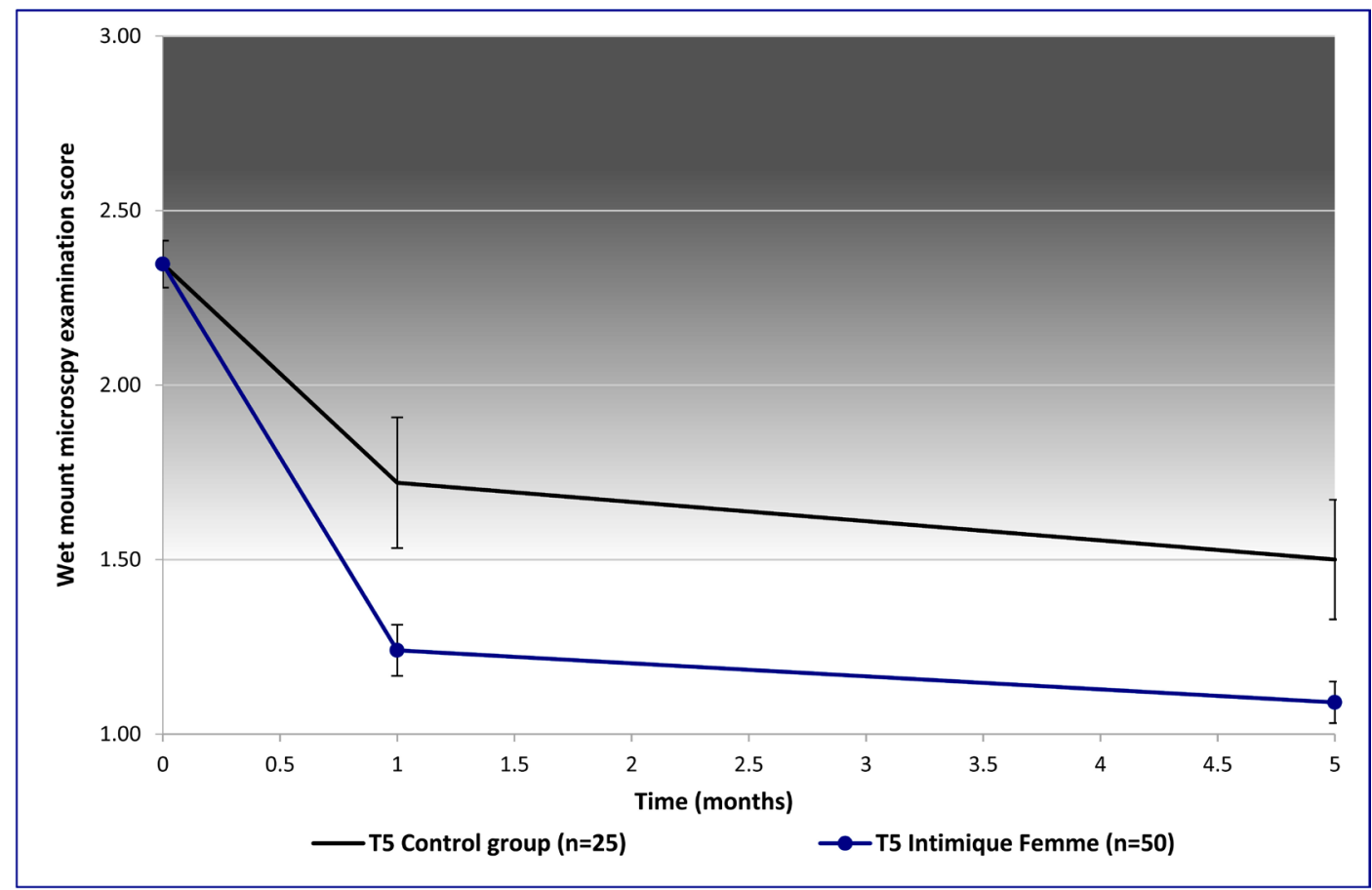

Figure 4. Vaginal wet mount microscopy examination score assessed at baseline, one month and five months after antibiotic therapy. Score 1: Lactobacillus dominated; 2: copresence of Lactobacilli and anaerobic Gram-negative bacteria; 3: presence of $\mathrm{BV}$-associated bacteria. Blue line: active group. Black line: control group. Mean values \pm standard error. 
overgrowth of pathogenic bacteria are associated with the development of BV.

We examined effects of the standard antibiotic treatment together with a new oral probiotic supplement on recurrences of BV, observed as clinical symptoms of vaginal infection and disturbances in the composition of vaginal microbiota. In our study, the recurrence rate of BV after treatment with Intimique ${ }^{\circledR}$ Femme was $16 \%$, significantly lower than the $40 \%$ presented in the control group and in the medical literature. It is consensual that BV involves the presence of a dense, structured and polymicrobial biofilm, primarily constituted by Gardnerella vaginalis clusters, strongly adhered to the vaginal epithelium. Our study protocol focused on demonstrating that the use of the probiotic Intimique ${ }^{\circledR}$ Femme preparation, simultaneously with standard treatment for BV, reduced the rate of recurrences, assessed using clinical and microbiological criteria, versus the standard treatment alone.

The typical bacteria with probiotic features include a diverse spectrum of Lactobacillus spp. (including L. rhamnosus, L. casei, L. acidophilus and L. plantarum) and Bifidobacterium spp. (including B. breve, B. bifidum, B. infantis and $B$. animalis), which colonize the healthy human gastrointestinal tract [13].

In order to positively impact on vaginal health, probiotic bacteria first need to colonize the female genital tract. For a successful colonization and to confer a health benefit to the host, the bacteria need to fulfill various criteria including: adherence to vaginal epithelial cells, production of hydrogen peroxide, bacteriocins and short chain fatty acids, restoration of vaginal $\mathrm{pH}$, and inhibition of potential pathogens associated with BV [14].

Adjuvant therapy after metronidazole or clindamycin treatment with $L$. acidophilus, L. casei rhamnosus (Lcr35) or Lactobacillus rhamnosus GR-1 and $L$. reuteri $\mathrm{RC}-14$ (GR-1/RC-14) is reported to provide better cure rates of symptomatic BV and diminish the frequency of recurrences during $1-6$ months follow up [15] [16] [17].

The new product tested in our study contains Lactobacillus plantarum PBS067, Lactobacillus rhamnosus LRH020 and Bifidobacterium animalis spp. lactis BL050, all constituents that have important effects on the main mechanism involved in the pathogenesis of BV.

Different studies demonstrated the presence of pili in Lactobacillus rhamnosus and L. plantarum and their involvement in adhesion [18], which could also be related to the biofilm formation. Beneficial biofilm formation by Lactobacillus strains is a valuable characteristic that can favor bacterial colonization when a probiotic product is administered to the host. Moreover, Lactobacillus rhamnosus LRH020 and Lactobacillus plantarum PBS067 have been found to secrete species-specific bacteriocins (rhamnocin and plantaricin) which have been demonstrated to exert a strong-antimicrobial capacity.

It is interesting to notice that Bifidobacterium spp., also contained in our product, can produce acetic acid that reduces bacterial and yeast growth, which may be a reason why it is contained in some vaginal probiotics, although these species are much less common in the female genital tract than Lactobacillus spp. 
[19]. Bifidobacterium animalis spp. lactis BL050 was also selected because, in particular conditions, it showed to self-produce group-B vitamins, which are a mandatory substrate for the growth of beneficial bacteria: its activity may have a booster effect on the colonization and proliferation of other friendly species in the gut first and then in the vaginal tract. Indeed, Bifidobacteria species generally exert bifidogenic effect, by promoting the growth of other lactic acid-producing bacteria and are responsible for controlling systemic inflammation.

In a previous study, we have demonstrated that our oral food supplement Intimique Femme is effective in colonizing the vaginal epithelium and that both the single strains and their combination can inhibit a wide panel of harmful pathogens, responsible for several vaginal infections [20].

The same multispecies probiotic supplement was also demonstrated to positively interact with the resident gastro-intestinal bacteria and colonize the large intestine at long-term by also improving intestinal symptoms in subjects affected by IBS-like constipation [21].

The current study focused on demonstrating that the use of Intimique Femme in the treatment of BV not only reduces the rate of relapses, but it also improves related symptoms and microbiological parameters of vaginal microbiota.

From a clinical point of view, since metronidazole, the most commonly prescribed antibiotic to treat BV, causes only transient suppression of the Gardnerella vaginalis populations in the vagina [22], the combined therapy with this antibiotic and Intimique ${ }^{\circledR}$ Femme can successfully reduce the anaerobic bacterial pathogens responsible for BV, delaying or preventing relapses and vaginal infection recurrences, thus preventing the administration of antibiotics in repetitive courses. The increased success rate of the combined therapy can be strongly correlated with the relative microbiota composition at the end of the study. After the reintegration period ( $\mathrm{AB}$ and subsequent 14 days of probiotics), the recovery of Lactobacillus-dominated microbiota was similar in the two groups, while a strong difference was represented by the unchanged dysbiotic portion in the control group and a partial dysbiotic composition in the active group, when compared to basal condition. At the end of the observation period, it was evident that the dysbiotic features in the negative control have been maintained and are probably at the basis of the recurrences episodes. On the contrary, partially disrupted microbiota in the probiotic group had turned into an almost totally Lactobacillus-dominated one, which is in line with our recurrences outcome.

Only few cases of drop-outs were registered, due to BV relapses occurred in the reintegration period: we speculate they probably experienced a failure of the antibiotic treatment and can be considered non-responder at T1 subjects, since no case of abandon of the study happened for personal compliance reasons.

Our study showed certain limitations, such as the lack of a placebo treatment arm and the limited follow-up after end of treatment. However, recurrences rate in the negative control perfectly fitted with epidemiological data, showing the consistency of the sample size. In any case, this study strengthens the evidence 
supporting the use of new strain-specific oral probiotics with well demonstrated activity associated with the creation and maintenance of a vaginal biofilm that hinders the persistence of BV-related infections.

\section{Conclusion}

This study showed that, in case of BV diagnosis, the complementary treatment of a strain-specific probiotic complex after antibiotics prophylaxis, is mandatory to reduce potential recurrences and cyclic use of further antibiotics. The outstanding results obtained with an oral supplement and the design of the study suggests a cyclic approach for the maximization of efficacy, rather than a continuative and long treatment. This solution is in line with a consumer-oriented method aiming at the higher patient compliance and protocol observance. Based on the wide antimicrobial spectrum of the probiotics complex as well as the capacity to colonize both gastro-intestinal tract and its proximal sites such as vagina, Intimique ${ }^{\circledR}$ Femme deserves further investigations in the urogenital tract infections domain.

\section{Conflicts of Interest}

The authors declare no conflicts of interest regarding the publication of this paper.

\section{References}

[1] Mittu, B., Kaur, B. and Balgir, P.P. (2015) Bacterial Vaginosis. Journal of Clinical Microbiology, 4, 3.

[2] Allsworth, J.E. and Peipert, J.F. (2007) Prevalence of Bacterial Vaginosis: 2001-2004 National Health and Nutrition Examination Survey Data. Obstetrics \& Gynecology, 109, 114-120. https://doi.org/10.1097/01.AOG.0000247627.84791.91

[3] Bagnall, P. and Rizzolo, D. (2017) Bacterial Vaginosis: A Practical Review. Journal of the American Academy of Physician Assistants, 30, 15-21. https://doi.org/10.1097/01.JAA.0000526770.60197.fa

[4] Chen, H.M., Chang, T.H., Lin, F.M., et al. (2018) Vaginal Microbiome Variances in Sample Groups Categorized by Clinical Criteria of Bacterial Vaginosis. BMC Genomics, 19, 876. https://doi.org/10.1186/s12864-018-5284-7

[5] Heczko, P.B., Tomusiak, A., Adamski, P., et al. (2015) Supplementation of Standard Antibiotic Therapy with Oral Probiotics for Bacterial Vaginosis and Aerobic Vaginitis: A Randomised, Double-Blind, Placebo-Controlled Trial. BMC Women's Health, 15, 115. https://doi.org/10.1186/s12905-015-0246-6

[6] Swidsinski, A., Mendling, W., Loening-Baucke, V., et al. (2005) Adherent Biofilms in Bacterial Vaginosis. Obstetrics \& Gynecology, 106, 1013-1023.

https://doi.org/10.1097/01.AOG.0000183594.45524.d2

[7] Coste, I., Judlin, P., Lepargneur, J.P. and Bou-Antoun, S. (2012) Safety and Efficacy of an Intravaginal Prebiotic Gel in the Prevention of Recurrent Bacterial Vaginosis: A Randomized Double-Blind Study. Obstetrics and Gynecology International, 2012, Article ID: 147867. https://doi.org/10.1155/2012/147867

[8] Sha, B.E., Gawel, S.H., Hershow, R.C., Passaro, D., Augenbraun, M., Darragh, T.M., 
Stek, A., et al. (2007) Analysis of Standard Methods for Diagnosing Vaginitis: HIV Infection Does Not Complicate the Diagnosis of Vaginitis. Journal of Lower Genital Tract Disease, 11, 240-250. https://doi.org/10.2165/00003495-200059030-00005

[9] Cook, R.L., Redondo-Lopez, V., Schmitt, C., Meriwether, C. and Sobel, J.D. (1992) Clinical, Microbiological, and Biochemical Factors in Recurrent Bacterial Vaginosis. Journal of Clinical Microbiology, 30, 870-877.

[10] Donders, G.G. (2000) Treatment of Sexually Transmitted Bacterial Diseases in Pregnant Women. Drugs, 59, 477-485.

[11] Ya, W., Reifer, C. and Miller, L.E. (2010) Efficacy of Vaginal Probiotic Capsules for Recurrent Bacterial Vaginosis: A Double-Blind, Randomized, Placebo-Controlled Study. American Journal of Obstetrics \& Gynecology, 203, 120.e1-120.e6. https://doi.org/10.1016/j.ajog.2010.05.023

[12] Bradshaw, C.S., Morton, A.N., Hocking, J., et al. (2006) High Recurrence Rates of Bacterial Vaginosis over the Course of 12 Months after Oral Metronidazole Therapy and Factors Associated with Recurrence. The Journal of Infectious Diseases, 193, 1478-1486. https://doi.org/10.1086/503780

[13] Figueroa-González, I., Quijano, G., Ramírez, G. and Cruz-Guerrero, A. (2011) Probiotics and Prebiotics-Perspectives and Challenges. Journal of the Science of Food and Agriculture, 91, 1341-1348. https://doi.org/10.1002/jsfa.4367

[14] McLean, N.W. and Rostenstein, I.J. (2000) Characterization and Selection of a Lactobacillus Species to Re-Colonize the Vagina of Women with Recurrent Bacterial Vaginosis. Journal of Medical Microbiology, 49, 543-552.

https://doi.org/10.1099/0022-1317-49-6-543

[15] Ozkinay, E., Terek, M.C., Yayci, M., et al. (2005) The Effectiveness of Live Lactobacilli in Combination with Low Dose Oestriol (Gynoflor) to Restore the Vaginal Flora after Treatment of Vaginal Infections. BJOG: An International Journal of Obstetrics \& Gynaecology, 112, 234-240. https://doi.org/10.1111/j.1471-0528.2004.00329.x

[16] Petricevic, L. and Witt, A. (2008) The Role of Lactobacillus casei rhamnosus Lcr35 in Restoring the Normal Vaginal Flora after Antibiotic Treatment of Bacterial Vaginosis. BJOG. An International Journal of Obstetrics \& Gynaecology, 115, 1369-1374. https://doi.org/10.1111/j.1471-0528.2008.01882.x

[17] Martinez, R.C., Franceschini, S.A., Patta, M.C., et al. (2009) Improved Cure of Bacterial Vaginosis with Single Dose of Tinidazole (2 g), Lactobacillus rhamnosus GR-1, and Lactobacillus reuteri RC-14: A Randomized, Double-Blind, Placebo-Controlled Trial. Canadian Journal of Microbiology, 55, 133-138.

https://doi.org/10.1139/W08-102

[18] Lebeer, S., Verhoeven, T.L.A., Perea Vélez, M., Vanderleyden, J. and De Keersmaecker, S.C.J. (2007) Impact of Environmental and Genetic Factors on Biofilm Formation by the Probiotic Strain Lactobacillus rhamnosus GG. Applied and Environmental Microbiology, 73, 6768-6775. https://doi.org/10.1128/AEM.01393-07

[19] Picard, C., Fioramonti, J., Francois, A., Robinson, T., Neant, F. and Matuchansky, C. (2005) Review Article: Bifidobacteria as Probiotic Agents-Physiological Effects and Clinical Benefits. Alimentary Pharmacology \& Therapeutics, 22, 495-512. https://doi.org/10.1111/j.1365-2036.2005.02615.x

[20] Mezzasalma, V., Manfrini, E., Ferri, E., Boccarusso, M., Di Gennaro, P., Schiano, I., Michelotti, A. and Labra, M. (2016) Orally Administered Multispecies Probiotic Formulations to Prevent Uro-Genital Infections: A Randomized Placebo-Controlled Pilot Study. Archives of Gynecology and Obstetrics, 295, 163-172. 
https://doi.org/10.1007/s00404-016-4235-2

[21] Presti, I., D’Orazio, G., Labra, M., La Ferla, B., Mezzasalma, V., Bizzaro, G., Giardina, S., Michelotti, A., Tursi, F., Vassallo, M. and Di Gennaro, P. (2015) Evaluation of the Probiotic Properties of New Lactobacillus and Bifidobacterium Strains and Their in Vitro Effect. Applied Microbiology and Biotechnology, 99, 5613-5626. https://doi.org/10.1007/s00253-015-6482-8

[22] Mayer, B.T., Srinivasan, S., Fiedler, T.L., Marrazzo, J.M., Fredricks, D.N. and Schiffer, J.T. (2015) Rapid and Profound Shifts in the Vaginal Microbiota Following Antibiotic Treatment for Bacterial Vaginosis. The Journal of Infectious Diseases, 212, 793-802. https://doi.org/10.1093/infdis/jiv079 